\title{
Emotional Intelligence is Associated with Blood Level Glucose: A Brief Report
}

\author{
Leehu Zysberg* \\ Associate professor, Gordon College of Education, Haifa, Israel
}

\begin{abstract}
Received: February 14,2019; Accepted: February 19,2019; Published: March 01, 2019
*Corresponding author: Dr. Leehu Zysberg, Gordon College of Education, Haifa, Israel, 15A Nurit street 8, Haifa, Israel 34654,Tel: ++972-58-4877771;Email: leehuzysberg@yahoo.com / leehu@gordon.ac.il
\end{abstract}

\begin{abstract}
Preliminary evidence associate aspects of emotion regulation and effective management of emotion-driven behavior with health outcomes in general and diabetic outcomes in particular. This study examined this association in a sample of 90 adult type 1 diabetic patients from an outpatient clinic in Israel. They provided non identifying demographic data and took 2 measures of emotional intelligence (test and self-report format) while also reporting their mean blood level glucose from their glucometers. The results support the hypothesized association: Both measures of EI showed unique negative associations with mean blood glucose levels. The results are briefly discussed and possible implications are pointed out.
\end{abstract}

Keywords: Type 1 Diabetes; Emotional Intelligence; Emotion Regulation; Diabetes Mellitus; Blood Level Glucose

\section{Introduction}

Diabetes mellitus is a serious chronic condition that necessitates adaptation of life styles, routines and adherence to a strict and often unpleasant monitoring and medication regimen $[1,2]$. Such restrictive behavioral boundaries, and living in the shadow of a never ending, incurable health risk, take their toll on patients, and even more so in the case of type 1 diabetes, in which patients have to adapt at a relatively early age [3]. As a result, emotional distress was found to be both a negative outcome of having to live with the challenge of this chronic condition from a young age and a potential factor in adhering to treatment and lifestyle recommendations to maintain control over the condition $[4,5]$. It is therefore of added value to identify resources that can support coping with the challenges and help maintain healthpromoting behaviors that lead to well-managed type 1 diabetes.

While the literature is replete with evidence and emerging models of coping resources in samples of diabetic patients - they usually relate to environmental and social resources such as education, income and family support [6,7]. A smaller body of research addresses the question of individual level resources, identifying self-efficacy and religiosity, among others, as individuals resources associated with better diabetic care outcomes [8]. Recent evidence has linked the relatively novel concept of emotional intelligence (EI) with diabetic outcomes, among others. EI is still debated upon but one of the most popular definitions views the concept as an amalgam of non- cognitive abilities associated with the identification, integration, understanding and management of emotions to direct behavior in an effective manner (also referred to as ability-EI, see: 9). The underlying hypothesis is that EI is associated with effective emotional monitoring and management and hence allows individuals to perform better under emotional duress. As a result individuals may be able to recruit resources to effectively meet the demands and challenges of their condition, adhere to care and monitoring procedures and maintain a healthier life style resulting in better indicators of diabetic balance and management [10]. While preliminary evidence support this hypotheses, further evidence is required to solidify the evidence associating EI and diabetic outcomes.

The current study sets out to test the potential associations between two measures of EI and a prominent and popular indicator of diabetic management: mean blood glucose level [11]. It was hypothesized that EI will show a negative association with blood glucose levels, while controlling for potential intervening factors such as age, education, religious (also cultural) affiliation and gender.

\section{Method}

\section{Settings and Sample}

The sample for this study was recruited from a community diabetes inpatient clinic, specializing in type 1 diabetes monitoring and treatment, in 2014. The clinic is located in a large urban center in northern Israel. We recruited only adult patients capable of providing informed consent. From 100 potential patients that were approached, 90 consented and provided data that allowed analysis, the others citing lack of time of being too distracted or busy to participate.

The final sample, then, included 90 (37 women and 53 men) patients. Their mean age was $40.12(\mathrm{sd}=7.49)$, they had an average of 14 years of formal education ( $\mathrm{sd}=3.55)$. The majority were Jewish (66\%), followed by Muslims (23\%), Christians (7\%) and Druze (4\%). They were all diagnosed with type 1 diabetes and were under regular follow up and treatment in the said clinic.

\section{Measures and Instruments}

Emotional intelligence Was assessed by two separate measures, representing 2 approaches to the assessment of EI - 
ability test format and self-report questionnaire. The literature on EI is torn between two traditions of measuring the concept, one influenced by the history of ability testing and the other from personality assessment [12]. To address this discrepancy we used two measures of ability EI:

Schutte emotional intelligence scale (SEIS) is a self-report measure, containing 33 items thematically associated with the content domains of ability-EI definitions. The measure is widely used in the field and has shown adequate reliability and validity [13].

Audio visual test of emotional intelligence (AVEI) is a 27 item audio visual test of emotion identification and analysis of complex emotional situations, two of the 4 facets of ability EI. The computer based test displays pictures and brief video clips of social interactions and asks the respondents to identify the emotions individuals are experiencing in each. The test has shown adequate reliability and predictive validity in educational and health related settings $[14,15]$.

Mean Blood Glucose levels were retrieved from the participants' glucometers at the time of data collection. This measure is considered an effective indicator of short term effectiveness of diabetic management $[10,11]$.

Demographic data including gender, age, formal education(in years), and religious/ cultural affiliation were also reported by the participants at the time of data collection.

\section{Procedure}

After obtaining IRB approval of the procedure at the author's institute, trained research assistants visited the clinic during its standard opening hours within a period of 2 weeks in summer 2014. They presented the study as a "study of various characteristics of type 1 diabetic patients" and asked potential participants to consent and provide the data described above. Participants filled out the questionnaires and took the AVEI test on laptops carried by the RAs. Completion of the procedure took about 20-25 minutes and took place in the waiting rooms at the clinic while waiting for an appointment or right after it. Participants did not receive any compensation for participation.

\section{Results}

Before testing the research hypothesis, descriptive statistics were calculated for the main study variables. Table 1 summarizes the main descriptive statistics for the sample:

The results suggest reasonable distributions of the variables with no floor or ceiling effects. Reliability is acceptable and allows continuation of parametric statistics.

Next, zero-order correlations were calculated to explore potential association patterns among the study variables. These are summarized in Table 2.

The preliminary results provide initial support for the study hypothesis: The two measures of EI associated negatively with mean blood glucose levels. Education levels showed a mild negative association with the same outcome and a strong positive association with AVEI. Surprisingly no correlations were found between the two measures of EI.

Next, to test the hypothesis positing that the two measures of EI will show negative associations with mean blood glucose levels, controlling for demographic background variables a multiple linear regression was used. The results are summarized in Table 3.

The results support the hypothesis and suggest that both measures of EI contribute separately to the explained variance in mean blood glucose levels while none of the background variables showed unique association with the target measure.

Table 1: Descriptive statistics for the study variables $(n=90)$

\begin{tabular}{|c|c|c|c|c|c|}
\hline & mean & sd & minimum & maximum & 3.21 \\
\hline SEIS* & 1.99 & 0.44 & 1.03 & 21 \\
\hline AVEI & 14.33 & 3.46 & 5 & 0.86 \\
\hline Mean glucose & 237 & 65.7 & 113 & 390 \\
\hline
\end{tabular}

Table 2: Pearson's zero-order correlations among the study variables $(n=90)$

\begin{tabular}{|c|c|c|c|c|c|c|}
\hline & & 1 & 2 & 3 & 4 & 5 \\
\hline 1. & M. Glucose & -- & & & & \\
\hline 2. & Age & -0.12 & -- & & & \\
\hline 3. & Education & $-.15^{*}$ & 0.01 & -- & & \\
\hline 4. & SEIS & $-.33^{* *}$ & 0.02 & -0.06 & -- & \\
\hline 5. & AVEI & $-.29 * *$ & -0.12 & $.52^{* *}$ & -0.05 & -- \\
\hline \multicolumn{7}{|c|}{$* \mathrm{p}<.05 * * \mathrm{p}<.01$} \\
\hline \multicolumn{7}{|c|}{ Note: SEIS grades were reversed for the sake of simplicity of presentation } \\
\hline
\end{tabular}




\begin{tabular}{|c|c|c|}
\hline \multicolumn{3}{|c|}{ Table 3. Summary of multiple regression analysis } \\
\hline & Beta & t-value \\
\hline Gender & -0.08 & -0.66 \\
\hline Age & -0.16 & -1.44 \\
\hline Education & -0.04 & -0.33 \\
\hline Religion & 0.06 & 0.51 \\
\hline SEIS & -0.29 & $-2.72^{* *}$ \\
\hline AVEI & -0.28 & $-2.49^{* *}$ \\
\hline${ }^{*} \mathrm{p}<.05{ }^{* *} \mathrm{p}<.01$ & & \\
\hline Total r-square $=.32 ; \mathrm{p}<.01$ & & \\
\hline
\end{tabular}

\section{Discussion}

The current study joins recent preliminary evidence associating EI and diabetic outcomes. Its results support the hypothesis that individuals' ability to identify, process and manage emotions in an effective manner negatively associates with mean blood level glucose (a short term indicator of effective management of diabetes). These and other similar results underline the emotional-motivational aspect of effective management of diabetes $[15,16,17]$. As a long term chronic condition it seem like patients need to overcome aversion to unpleasant blood tests, excluding certain foods and beverages from their diet, and lead a life under constant health threat [16] EI may serve as an individual resource to allow more effective management of these elements and thus reach better glycemic management and better health outcomes in diabetic patients.

The results of this study also reveal patterns that are somewhat surprising and shed a new light on what we may or may not yet know about the nature of EI and its role in effective diabetic management: The most interesting finding perhaps is the fact that in the regressions analysis no demographic variable entered the model, despite what one may expect from the literature $[7,8]$. The fact that once EI was entered into the regression, other background variables no longer showed associations with blood glucose levels may actually be encouraging: it may indicate that these individual resources override personal factors such as age, income or education level.

Another interesting finding is what the lack of association between the two measures of EI in combination with the unique contribution of both measures to the regression model may suggest. While both measures of EI are derived from the ability model of EI, the different modes of assessment used (testing vs. Self-report questionnaires) may actually tap different aspects of the general concept of EI [18]. The methodological literature speaks of the unique characteristics and inherent biases in different measurement modes $[19,20]$. While this may sound at first like bad news, the fact that both measures provided unique added value in accounting for our outcome measures, suggests that using different measures of the same concept may actually increase the ability to better understand and account for the outcomes and may represent a broader sample of the content one is trying to assess or measure.

\section{Conclusion}

However, interpretation of these results should be done with care, considering the study limitations: Our sample is relatively modest in both size and representativeness. Culture, health system characteristics and other factors may modify the effects found here and thus should be considered in interpreting these results. Measures of EI vary and alternative models of EI are offered in the literature and should be tested alongside the currently used one in future studies. Last but not least - type 1 diabetes patients comprise a minority in the population of diabetic patients and these results should be tested in samples of type 2 diabetes patients.

Limitations notwithstanding, this study offers further evidence to support the budding literature on the role EI may play in diabetic management and serve as a basis for further development of screening tools as well as interventions that may help foster resilience, adherence with care protocols and as a result better quality of life and longevity among diabetic patients.

\section{References}

1. Hernandez CA. Integration: the experience of living with insulin dependent (type 1) diabetes mellitus. The Canadian journal of nursing research= Revue canadienne de recherche en sciences infirmieres. 1996;28(4):37-56.| Doi: 10.1177/014572179502100106

2. Siddiqui MA., Khan, M. F., \& Carline, T. E. Gender differences in living with diabetes mellitus. Materia socio-medica. 2013;25(2):140] Doi: 10.5455/msm.2013.25.140-142

3. Van Bastelaar KM, Pouwer F, Geelhoed-Duijvestijn PHLM, Tack CJ, Bazelmans E, Beekman AT, et al. Diabetes-specific emotional distress mediates the association between depressive symptoms and glycaemic control in Type 1 and Type 2 diabetes. Diabetic Medicine. 2010;27(7):798-803.' Doi: 10.1111/j.1464-5491.2010.03025.x

4. Polonsky WH, Fisher L, Earles J, Dudl RJ, Lees J, Mullan J \& Jackson RA. Assessing psychosocial distress in diabetes: development of the diabetes distress scale. Diabetes care. 2005;28(3):626-631.]

5. Strandberg RB, Graue M, Wentzel-Larsen T, Peyrot M \& Rokne B. Relationships of diabetes-specific emotional distress, depression, anxiety, and overall well-being with HbA1c in adult persons with type 1 diabetes. Journal of psychosomatic research. 2014;77(3):174-179. Doi: 10.1016/j.jpsychores.2014.06.015

6. Grey M, Davidson M, Boland EA \& Tamborlane WV. Clinical and psychosocial factors associated with achievement of treatment goals in adolescents with diabetes mellitus. Journal of adolescent health. 2001;28(5):377-385.

7. Nam S, Chesla C, Stotts NA, Kroon L \& Janson SL. Barriers to diabetes management: patient and provider factors. Diabetes research and clinical practice. 2011;93(1):1-9., Doi: 10.1016/j.diabres.2011.02.002

8. Gao J, Wang J, Zheng P, Haardörfer R, Kegler MC, Zhu Y et al. Effects of self-care, self-efficacy, social support on glycemic control in adults with type 2 diabetes. BMC family practice. 2013;14(1):66.| Doi: 10.1186/1471-2296-14-66

9. Cobb CD, \& Mayer JD. Emotional Intelligence: What the Research Says. Educational leadership. 2000;58(3):14-18. 
10.Zysberg L. Diabetic management and emotional intelligence-an emerging direction in current research. JEndocrinol Diab. 2016;3(2):13. Doi: 10.15226/2374-6890/3/2/00145

11. Maran A, Crepaldi C, Tiengo A, Grassi G, Vitali E, Pagano G et al. Continuous subcutaneous glucose monitoring in diabetic patients: a multicenter analysis. Diabetes Care. 2002;25(2):347-352.]

12. Brackett MA, Rivers SE, Shiffman S, Lerner N, Salovey P. Relating emotional abilities to social functioning: a comparison of selfreport and performance measures of emotional intelligence. Journal of personality and social psychology.2006;91(4):780-795. Doi: 10.1037/0022-3514.91.4.780

13. Schutte NS, Malouff JM, Hall LE, Haggerty DJ, Cooper JT, Golden CJ, et al. Development and validation of a measure of emotional intelligence. Personality and individual differences. 1998;25(2):167-177. Doi: 10.1016/S0191-8869(98)00001-4

14.Zysberg L, Levy A, \& Zisberg A. Emotional intelligence in applicant selection for care-related academic programs. Journal of Psychoeducational Assessment. 2011;29(1):27-38.] Doi: 10.1177/0734282910365059

15.Zysberg L, Bar Yoseph T, \& Goldman M. Emotional intelligence and glycemic management among type I diabetes patients. Journal of health psychology. 2017;22(2):158-163.| Doi: 10.1177/1359105315596373
16.Zysberg L, Lang T, Zisberg A. Parents' emotional intelligence and children's type I diabetes management. Journal of health psychology. 2013;18(9):1121-1128. Doi: 10.1177/1359105312459097

17. Housiaux M, Luminet O, Van Broeck N, Dorchy H. Alexithymia is associated with glycaemic control of children with type 1 diabetes. Diabetes \& metabolism. 2010;36(6):455-462.] Doi: 10.1016/j. diabet.2010.06.004

18. Brackett MA, \& Mayer JD. Convergent, discriminant, and incremental validity of competing measures of emotional intelligence. Personality and social psychology bulletin. 2003;29(9):1147-1158.] Doi: $10.1177 / 0146167203254596$

19. Brackett MA, Rivers SE, Shiffman S, Lerner N, Salovey P. Relating emotional abilities to social functioning: a comparison of selfreport and performance measures of emotional intelligence. Journal of personality and social psychology. 2006;91(4):780.] Doi: 10.1037/0022-3514.91.4.780

20. O'Connor Jr RM, Little IS. Revisiting the predictive validity of emotional intelligence: Self-report versus ability-based measures. Personality and Individual differences. 2003;35(8):1893-1902.| Doi: 10.1016/ S0191-8869(03)00038-2 